\title{
Capacidades institucionales para la vinculación científico- tecnologica en una universidad de gestión estatal
}

\author{
Di Meglio Fernanda ${ }^{1}$ \\ ${ }^{1}$ Universidad Nacional del Centro de la Provincia de Buenos Aires
}

Recibido: 10 de octubre de 2016

Aceptado: 10 de diciembre de 2017

\begin{abstract}
Resumen
Desde una perspectiva teórica proveniente de la ciencia política, en este artículo se identifican y analizan las distintas capacidades institucionales universitarias implicadas en el desarrollo de la política de vinculación científico-tecnológica en una universidad argentina de gestión estatal. Los datos empíricos en los que se basa esta investigación se generaron a través de análisis documental proveniente de la universidad objeto de estudio y se complementó con entrevistas semiestructuradas aplicadas a distintos funcionarios vinculados a la temática. Los resultados obtenidos muestran que la universidad estudiada desarrolló capacidades institucionales vinculadas a la creación de una estructura de interacción ad hoc que cumple la función de enlace entre el entorno y la universidad, a la implementación de una política de incentivos para promover la participación de la comunidad
\end{abstract}

Palabras claves: capacidades institucionales, vinculaciones científico-tecnológicas, universidad pública

\begin{abstract}
From a theoretical perspective from political science, this article identifies and analyzes the various institutional capacities involved in the development of policy linkage in a state-run university Argentina. The empirical data on which this study is based were generated through documentary analysis from the university under study and supplemented with semi-structured interviews applied to various officials in the subject. The results show that the university developed institutional capacities linked to the creation of a structure of ad hoc interaction that serves as the link between the territory and the university, and the implementation of a policy of incentives to promote community participation academic in these activities as well as the involvement of the university
\end{abstract}


in public and private spaces and the active leadership of the university managers responsible for this function.

Key words: institutional capacities, linking scientific and technological, public university.

\section{Introducción}

La mayoría de los trabajos en la temática señalan las dificultades que enfrentan las universidades para vincularse con los sectores productivos (Bozeman, 2000; Fernández Di Lucio, 2000; D’Este y Patel, 2007; De Fuentes y Dutrénit, 2014). En particular, el peso de las actividades de vinculación y transferencia hacia el sector productivo resulta escasa en relación al resto de las actividades que llevan adelante las universidades argentinas (Chudnovsky y López, 1996; Elzinga, 1997; Versino, 2012). Dicha situación ha despertado una preocupación generalizada, debido al importante rol que este tipo de actividades tiene sobre los procesos de innovación y desarrollo económico (Clark, 1998; Leydesdorff y Etzkowitz, 1996). En este marco, se implementaron distintos mecanismos institucionales con el objetivo de fortalecer las interrelaciones entre éstos actores (Dagnino, Thomas y Davyt, 2000; Sutz, 2000) e incrementar el peso de estas actividades en las universidades (Chudnovsky y López, 1996; Elzinga, 1997; Versino, 2012). Sin embargo, las dificultades inherentes a este proceso sigue siendo uno de los puntos de preocupación en la mayoría de los países. Como ejemplo de lo anterior se encuentra el estudio de Bozeman (2000) llevado a cabo en EE.UU, el cual explora la influencia que tiene la agenda de investigación para el desarrollo de actividades de vinculación en las universidades y los centros de investigación norteamericanos. En Inglaterra, la investigación realizada por D’Este y Patel (2005) explora como los investigadores universitarios interactúan a lo largo de una variedad de canales con la industria, mostrando que dichos canales no tienen en gran parte relación entre sí, y que la frecuencia de la interacción varía significativamente en función de las disciplinas científicas. Por otra parte, el estudio expresa la importancia distintiva de los factores asociados a este fenómeno en tres diferentes niveles de agregación: el nivel de la universidad, el departamento y el investigador universitario.

Por su parte, Solleiro, Ritter y Escalante (2000) en Brasil y Casalet y Casas (1998) en México abordan distintos aspectos de la vinculación teniendo en cuenta las características propias de las universidades latinoamericanas. En este sentido, es importante destacar que la colaboración 
universidad-sector productivo en América Latina se lleva a cabo bajo circunstancias especiales que se derivan del estado de desarrollo económico, tecnológico, empresarial, social y político de los diferentes países. Estas circunstancias determinan ciertas características distintivas en las relaciones que las diferencian de experiencias similares en otros países, especialmente del mundo desarrollado. En este marco, Solleiro et al., (2000) y Casalet y Casas (1998:23) exponen los limitantes institucionales que impiden una mayor vinculación en la región, entre los cuales se encuentra: la falta de políticas y normativa adecuada, la inexistencia de una infraestructura apropiada para el desarrollo de la investigación científica y tecnológica y la escasa cultura y disposición de los académicos universitarios para vincularse con el sector productivo. La condición en Argentina no es diferente a la descripta arriba. Aunque existen pocos estudios dedicados a medir las actividades de vinculación en las universidades argentinas, estudios como el de Estébanez y Korsunsky (2004) reportan una escasa propensión de las universidades argentinas a realizar actividades de vinculación en el marco de sus proyectos de investigación, y estudios más recientes (Carullo, 2009) exponen que la mayoría de las actividades están principalmente asociadas a cuestiones de formación más que a actividades vinculadas a la incubación de empresas, el desarrollo de proyectos de investigación o desarrollo o bien la prestación generalizada de servicios tecnológicos. A pesar de la baja performance expuesta, existen escasos estudios en Argentina enfocados en analizar los procesos que se desarrollan al interior de las instituciones universitarias y en su relación con la sociedad, por lo tanto, este trabajo busca identificar- a través de un estudio de caso- las diversas capacidades que poseen o que deben construir las universidades para poner en práctica sus políticas de vinculación científico-tecnológicas. El artículo está organizado en seis secciones: en la primera se presenta el análisis de literatura especializada donde se identifican los principales estudios en la temática; en la siguiente sección se aborda el concepto de "capacidades institucionales universitarias" y como este elemento puede ser útil para explicar los procesos que se desarrollan al interior de las instituciones universitarias; en la tercera se describe el método de investigación utilizado en el presente estudio; en la cuarta sección se exponen los resultados encontrados; en la quinta y sexta secciones se realiza una discusión de los resultados, y se exponen las conclusiones. 


\section{Antecedentes bibliográficos}

\section{Normatividad institucional y factores técnicos-administrativos}

Uno de los aspectos que aparecen abordados en una gran cantidad de estudios son aquellos referidos a los aspectos normativos e institucionales de las universidades (Fernández et al., 2010; Kababe, 2010; Casalet, 2010; Solleiro, 2014). Por ejemplo, autores como Fernández, Castro, Conesa y Gutiérrez, (2010) y Solleiro (2014) ponen el énfasis en los marcos normativos que facilitan las relaciones y en las políticas de remuneración y normatividad administrativa en el interior de las organizaciones. Por otro lado, autores como Casalet (2010) y Kababe (2010) se han centrado en las estructuras organizacionales para promover la vinculación, que si bien se han generalizado en la mayoría de las universidades, todavía es común encontrar organizaciones que no cuentan con la estructura institucional adecuada. Hay muchos casos en los que se intenta sustentar una estrategia de vinculación mediante el esfuerzo personal de los académicos, quienes, por lo general, no tienen la experiencia ni el tiempo disponible para este tipo de actividades. Otras instituciones han creado oficinas especializadas que centralizan las actividades de vinculación, pero no las han dotado de personal calificado en los temas fundamentales. Es sumamente difícil encontrar personal de este tipo, dado que la gestión tecnológica es una disciplina novedosa, sobre todo en el contexto latinoamericano (Solleiro, 2014). En el estudio que realiza Kababe (2010) se señalan debilidades estructurales y organizativas, sobre todo dificultades en la trama de relaciones formales y se evidencia insuficiente reflexión sobre la complejidad que supone llevar a cabo estas actividades.

\section{Condiciones del contexto y modalidades de interacción}

Otros estudios muestran la importancia que tienen las condiciones del contexto para las relaciones entre las instituciones universitarias y los sectores productivos. Un ejemplo, es el estudio realizado por Solleiro et al. (2000) que señala que la colaboración universidad-sector productivo se lleva a cabo bajo circunstancias especiales que derivan del estado de desarrollo económico, tecnológico, empresarial, social y político de los diferentes países. Estas circunstancias determinan ciertas características distintivas en las relaciones que las diferencian de experiencias similares en otros países, especialmente del mundo desarrollado. En el mismo trabajo, los autores mencionan 
que estas circunstancias particulares se expresan como importantes limitantes para la vinculación que representan un verdadero reto para su adecuada gestión. Con relación a lo anterior, Rubiano, Rangel y Cuca (2013) encontraron que a veces los sectores productivos no tienen la capacidad suficiente para absorber e implementar el conocimiento, especialmente cuando se refiere a empresas pequeñas o medianas, las cuales necesitan primero mejorar sus procesos con la ayuda de la investigación. Sin embargo, así como hay elementos externos que las universidades no puede controlar y que dificultan sus procesos de vinculación, hay otros elementos que la favorecen. En el mismo trabajo de Rubiano et al. (2013), los autores mencionan la importancia que tiene la existencia de un marco político adecuado en educación y en investigación y desarrollo (I+D) que permita generar y consolidar los procesos de vinculación. En particular, hay que destacar que existe una proliferación de trabajos que abordan distintas problemáticas vinculadas a este fenómeno, sin embargo la mayoría de ellos no trascienden las meras descripciones de casos específicos (Versino, 2012). Si bien, dichos estudios son de importancia para dar cuenta de esta función no contribuyen a comprender la dimensión y las condiciones de funcionamiento del fenómeno en el ámbito local (Versino, 2012). Asimismo, es importante destacar que la mayoría de estos estudios analizan universidades de tamaño grande ${ }^{12}$. Los análisis ponen especial énfasis a distintos elementos internos y externos del proceso de vinculación de las universidades, sin embargo, estos aspectos siempre fueron analizados de forma separada, por lo tanto, la propuesta pretende aportar un enfoque analítico integrado que nos permita analizar estos elementos de manera conjunta.

\section{La vinculación de las universidades y el concepto de "capacidades estatales"}

Investigadores relacionados a la sociología y a la ciencia política consideran que el concepto de "capacidades estatales" es una importante herramienta analítica para la comprensión del estado y su relación con la sociedad; es decir, la encuentran útil para explicar los procesos que se desarrollan al interior de las instituciones estatales. Debido a lo anterior, en esta sección se abordará el concepto de "capacidades estatales" como una herramienta analítica alternativa para explorar la política de vinculación científico-tecnológica pero, además como una alternativa de abordaje de la gestión de este tipo de políticas.

${ }^{12}$ Universidad de Buenos Aires, Universidad Nacional de la Plata, Universidad de Córdoba. 


\section{Justificación del concepto de "capacidades estatales" en esta investigación}

La importancia de hablar sobre "capacidades estatales" es su función como herramienta analítica que sirve para explicar las diversas capacidades que poseen o que deben construir los actores estatales para poner en práctica sus políticas y acciones (Sckopol, 1985). Una de las acepciones generales es aquella que considera a la capacidad estatal como "la habilidad por parte de las agencias estatales para realizar tareas con efectividad, eficiencia y sustentabilidad" (Hildebrand y Grindle, 1997 citado por Alonso, 2007:18). En el caso particular de esta investigación, el concepto de "capacidades institucionales" se utiliza para analizar cómo la universidad "genera" políticas de vinculación" ${ }^{3}$, ya que, como señala Sckopol, "los estudios más fructíferos de las capacidades institucionales tienden a centrarse en áreas de actuación políticas concretas" (1985:25) que, para los fines de esta propuesta, se denominarán "capacidades institucionales universitarias". En ese sentido, es que esta concepción será considerada en el marco del análisis sobre la vinculación de las universidades públicas.

\section{Sobre el concepto de capacidades estatales}

Dentro del concepto de capacidades estatales deben considerarse, dos dimensiones que están presenten: por un lado, lo que refiere a las capacidades técnico-administrativas y, por otro, una dimensión relacional que se refiere a la relación de la institución estatal con el entorno socioeconómico. Desde esta perspectiva, el análisis de capacidades prioriza un mirada desde adentro de la organización estatal, mientras que la dimensión relacional pone el foco en el nexo institución-sociedad (Alonso, 2007). En este sentido, el marco conceptual nos permite analizar dos aspectos: las características institucionales de las universidades, entre las que se encuentran por un lado las estructuras institucionales y sus funciones (dimensión institucional) y la relación universidad-sociedad (dimensión relacional). Este abordaje permite un análisis tanto de los aspectos internos de la universidad, indagando sobre los mecanismos de enlace y sobre los factores técnico-administrativos y organizacionales como a su vez en los aspectos externos relacionados a la dimensión relacional y contextual que que potencian o dificultan la relación entre estos actores.

${ }^{13}$ En este sentido, cabe destacar que se trata de instituciones autónomas, ya que cuentan con "la facultad del autogobierno, la decisión de políticas internas, la distribución de sus propios recursos, la determinación de los objetivos de su actividad" (Vaccarezza, 2006: 35). 
En este sentido, también se incorpora la variable contextual o contexto de acción propuesta por Hilderbrand y Grindle (1994) referido al entorno económico, social y político en que "las organizaciones intentan llevar adelante sus actividades y el alcance en que las condiciones del ambiente de acción facilitan o limitan su desempeño" (Hilderbrand y Grindle, 1994:17). Bajo este marco, se sostiene como hipótesis general que "consolidar una relación más efectiva entre las universidades y los sectores productivos está relacionado con determinado contexto en el que actúa la universidad, con una relación particular que establece con su entorno y con características organizacionales específicas.”

Síntesis de las dimensiones a analizar bajo el concepto de "capacidades institucionales universitarias".

\section{Dimensión organizacional}

En relación a la primera dimensión, de las capacidades técnico-administrativas u organizacionales, se indagará sobre la organización interna de la universidad en relación a las políticas de vinculación científico-tecnológica. En este marco, se analizará las áreas encargadas de la función (las estructuras de interacción), los incentivos propuestos y la profesionalización y capacidad de los recursos humanos como así también la orientación estratégica de la universidad en relación a esta función (Clark, 2004). En principio, hay que destacar que para la puesta en marcha de una política de vinculación científico-tecnológica todas las universidades crearon oficinas coordinadoras de toda la actividad de la institución como así también estructuras de interacción ad hoc como las fundaciones. En relación a las estructuras de interacción Clark (2004) se refiere a las unidades de apoyo orientadas a atender las necesidades de los agentes internos y externos. La existencia de estas estructuras constituye uno de los elementos más visible del compromiso de la universidad con esta función (Feldman, Feller; Bercovitz y Burton, 2002). Sin embargo, su sola existencia no garantiza una relación efectiva con el entorno (Lockett, Wrigth y Franklin, 2003; Siegel Waldman y Link, 2003). En este sentido, es importante evaluar las competencias del personal y su experiencia para desarrollar actividades de este tipo (Jensen, Thursby y Thursby, 2003; Siegel et al., 2003). Otro de los aspectos es evaluar la existencia de políticas de adaptación a las nuevas condiciones del entorno (planes institucionales) y los 
incentivos propuestos por cada universidad para desarrollar iniciativas de vinculación (Decter, Bennett y Leseure, 2007). Las oportunidades para la vinculación al medio productivo se encuentran en gran medida garantizadas por los recursos financieros que se implementan para el desarrollo de esta función como así también por los incentivos que se orientan a promover la participación de los investigadores en estas actividades.

\section{Dimensión relacional}

En cuanto al análisis de la universidad a través de su dimensión relacional, se analiza el entramado socio-cultural y la participación de la universidad en espacios públicos-privados de promoción de estas actividades. En esta línea Clark (1998, 2004) señala que para que se establezcan relaciones efectivas con el entorno es necesario que las universidades implementen mecanismos amplios y participativos promoviendo la articulación y la cooperación con otros actores involucrados en el proceso de vinculación. En este sentido, se tendrá en cuenta tres aspectos de la dimensión relacional: el tipo de relacionamiento que establece con los actores públicos y privados, la participación en redes y asociaciones públicos y privadas locales involucradas en la temática y los vínculos que establece con los distintos organismos especializados del plano políticoinstitucional provincial y nacional.

\section{Tabla 1}

Síntesis de las variables a analizar bajo el concepto de "capacidades institucionales universitarias"

\begin{tabular}{|c|c|c|c|}
\hline $\begin{array}{c}\text { Factor } \\
\text { Explicativo }\end{array}$ & Dimensiones & $\begin{array}{c}\text { Sub- } \\
\text { dimensiones }\end{array}$ & Indicadores \\
\hline \multirow{4}{*}{ 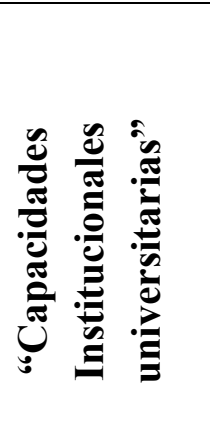 } & \multirow{4}{*}{$\begin{array}{c}\text { Dimensión } \\
\text { técnico- } \\
\text { administrativ } \\
\text { as u } \\
\text { organizaciona } \\
\text { les }\end{array}$} & \multirow{4}{*}{$\begin{array}{l}\text { Organización } \\
\text { Interna de la } \\
\text { Universidad }\end{array}$} & Organización estratégica \\
\hline & & & Incentivos a la vinculación \\
\hline & & & $\begin{array}{l}\text { Estructuras de interacción (Áreas } \\
\text { encargadas) }\end{array}$ \\
\hline & & & $\begin{array}{l}\text { Recursos Humanos (Dirección y } \\
\text { Liderazgo) }\end{array}$ \\
\hline
\end{tabular}




\begin{tabular}{|l|l|c|l|}
\hline \multirow{2}{*}{$\begin{array}{l}\text { Dimensión } \\
\text { Relacional }\end{array}$} & Entorno & $\begin{array}{l}\text { Tipo de relacionamiento con actores } \\
\text { públicos y privados }\end{array}$ \\
\cline { 3 - 3 } & $\begin{array}{l}\text { Participación en espacios públicos- } \\
\text { privados locales de promoción de } \\
\text { estas actividades }\end{array}$ \\
\cline { 3 - 3 } & $\begin{array}{l}\text { Vínculos con agencias locales, } \\
\text { provinciales y nacionales de } \\
\text { promoción científico-tecnológica }\end{array}$ \\
\hline
\end{tabular}

\section{Fuente: elaboración propia.}

Por último, se incorpora la variable contextual o contexto de acción propuesta por (Hilderbrand y Grindle, 1994). Entre los factores que se toman en cuenta para operacionalizar este entorno se incluyen, entre otros: los económicos (nivel y crecimiento de PBI, condiciones del mercado de trabajo); los políticos (liderazgo y legitimidad del gobierno, la movilización de la sociedad civil); y los sociales (nivel de desarrollo de recursos humanos, alcance de la movilización social y sus necesidades). Estos entornos poseen peculiaridades intrínsecas para desarrollar mecanismos singulares de promoción según sus condiciones específicas.

\section{Metodología}

La investigación optó por un estudio de caso cuyo objetivo general fue describir la experiencia reciente de articulación universidad-sector productivo desarrollada en una universidad argentina de tamaño mediano de la Provincia de Buenos Aires, utilizando una doble perspectiva. Por una parte, se propuso comprender la especificidad que asume la política y gestión universitaria de la vinculación desplegada en el caso estudiado, atendiendo a sus características organizacionales. Por la otra, pretendimos analizar el tipo de modelo universitario de vinculación a partir de las dimensiones pre-establecidas en el enfoque teórico desarrollado. De acuerdo con el diseño de la investigación que fue guiando la construcción de la evidencia empírica, la primera etapa se centró en identificar los elementos contextuales que caracterizan al entorno económico y social en donde se desenvuelve la universidad. En una segunda etapa, nos ocupamos en la formulación de las políticas y en los mecanismos institucionales de gestión de la vinculación desarrollada. En una tercera etapa nos ocupamos de investigar los aspectos externos vinculados a 
sus relaciones con el entramado político-institucional local, provincial y nacional. Finalmente, en una cuarta etapa analizamos los alcances y las limitaciones del actual modelo de vinculación desplegado por la institución académica estudiada. La naturaleza "multidimensional" del problema a investigar, nos llevó a utilizar una combinación de métodos y técnicas. Utilizando un número limitado de conceptos, se combinó la realización de entrevistas semi-estructuradas a gestores universitarios responsables de las actividades de vinculación con análisis de material documental resoluciones, convenios, informes institucionales, boletines informativos, folletos publicitarios- y estadístico sobre tales experiencias. Para cumplir con este esquema de trabajo, en el marco de la investigación se planteó como primera actividad decidir el caso en el cual realizaríamos el estudio. Para ello, se inició por la recopilación de información sobre las universidades de tamaño mediano de la Provincia de Buenos Aires ${ }^{14}$ dado que la mayoría de los estudios abordan universidades tamaño de tamaño grande ${ }^{15}$ y posteriormente se seleccionó la Universidad Nacional del Sur (UNS). Esta selección se justificó en varios aspectos: en primer lugar, porque la mayor parte de los análisis se concentran en las universidades de tamaño grande y por el importante peso que tiene la UNS en el conjunto de universidades de la Provincia de Buenos Aires. Tal es así, que esta universidad concentra el 10\% de la población estudiantil (24.546 estudiantes) y representa el 15,3\% de los docentes-investigadores (1266 docentes categorizados) en el total provincial (Anuario SPU, 2013). Además, la universidad seleccionada ocupa uno de los primeros puestos de las universidades con mayor vólumen de "recursos propios" (indicador aproximado de los vínculos con terceros) recibidos durante el año 2008 y 2009 en relación al resto de las universidades de gestión estatal de tamaño mediano (ocupa el lugar 3 de un total de 14 universidades). Además, el estudio se focaliza en esta universidad por motivos prácticos y por la accesibilidad a las fuentes, planteando de esta manera una investigación principalmente, por cuestiones de viabilidad de la propuesta, así como para mantener el realismo de las mediciones (Kish, 1995).

\section{Tendencias recientes en las universidades de gestión estatal argentinas}

En primer lugar, hay que destacar que para la puesta en marcha de una política de vinculación científico-tecnológica las universidades crearon oficinas coordinadoras de toda la actividad de la institución como así también estructuras ad hoc como las fundaciones. En

\footnotetext{
${ }^{14}$ Con excepción de aquellas asentadas en el denominado conurbano bonaerense.

${ }^{15}$ La clasificación de las universidades por tamaño está basada en la clasificación propuesta por la RedVitec que define las categorías según el presupuesto de las universidades.
} 
Argentina, dichas áreas no surgen en forma aislada, sino que se implementan en el contexto de la reforma de la educación superior acaecida a partir de los años noventa del siglo XX que coloca a esta problemática como uno de los nuevos desafíos de la universidad (Rama, 2006). Estas oficinas son un elemento importante para el desarrollo de la política de vinculación con el entorno, pues se trata de las estructuras que se encargan de la promoción y ejecución en sus estrategias de vinculación con el medio. Dichas oficinas pueden denominarse con diferentes nombres según la importancia y el papel que le otorga la institución. Puede nombrarse oficina, área, secretaría o subsecretaria, entre otros. Por su parte, las Secretarías o instancias similares, formalmente incluidas en la estructura de la universidad, por lo general funcionan bajo el ámbito de rectorado, y operan como las vías de salida más formales. Estas estructuras se articulan con las facultades, departamentos y grupos de investigación para funcionar como enlace con el medio. También las universidades cuentan con otro tipo de estructuras ad-hoc para el desarrollo de las actividades de vinculación, por lo general estas áreas cuentan con cierto grado de autonomía operativa. En el caso de las universidades argentinas, esta modalidad se verifica en fundaciones u otras organizaciones, que funcionan como entidades de apoyo y cuentan con mayores grados de libertad en lo referido a la captación y asignación de fondos adicionales. En este marco, en el siguiente apartado, se identificarán estos aspectos con el objetivo de identificar aquellos aspectos organizacionales presentes en la universidad seleccionada.

\section{Breve caracterización de la política de vinculación científico-tecnológica en la UNS}

La Universidad Nacional del Sur (UNS) -cuyo antecedente es el Instituto Tecnológico del Sur $^{16}$ - creada en 1956 se encuentra ubicada en la ciudad de Bahía Blanca, en el sudoeste de la Provincia y desde sus orígenes se caracterizó por desarrollar una activa política de vinculación dirigida a transferir el conocimiento producido en la universidad a los sectores productivos. Según Krotsch (1998) esta universidad llevó adelante una de las primeras experiencias de vinculación de las universidades argentinas a través de la creación del Programa de Investigación y Desarrollo del Complejo Petroquímico Bahía Blanca (PIDCOP) en 1977. En el marco este Programa se iniciaron actividades conjuntas de capacitación, investigación y desarrollo con las empresas integrantes del

\footnotetext{
${ }^{16}$ Fundada por Ley provincial en 1946, inició sus actividades bajo la dependencia académica de la Universidad Nacional de La Plata.
} 
Polo Petroquímico de Bahía Blanca, generando una estructura que promovió una relación dinámica entre los sectores académico, científico-tecnológico y productivo, impulsando la creación y transferencia de tecnología hacia el sector industrial. Posteriormente, la UNS mantuvo dicha política a partir del establecimiento de diversas estrategias de vinculación y la creación de instancias institucionales orientadas a tal fin.

\section{Dimensión contextual}

Aspectos socio-demográficos

Bahía Blanca fundada en año 1828 abarca $2.247 \mathrm{~km}^{2}$ y está ubicada geográficamente a 647 km de la Ciudad Autónoma del Buenos Aires en el sudoeste de la Provincia, limita con los partidos de Villarino, Tornquist, Coronel Pringles y Coronel Rosales, y es la ciudad más importante del sur de la provincia de Buenos Aires. De acuerdo con datos correspondientes al último Censo Nacional 2010 registra un total de 301. 531 habitantes. En particular, su rol estratégico en el modelo agroexportador "la convirtió en receptora de importantes inversiones en infraestructura productiva como ferrocarriles y puertos y la posicionó como cabecera de una región agropecuaria muy importante" (Municipalidad de Bahía Blanca, 2009). El sostenido ritmo de crecimiento registrado en la ciudad y las importantes funciones que allí se desarrollan "le otorgan la categoría de centro urbano regional" (Prieto 2008: 203). En este sentido, constituye un importante nodo de comunicaciones y transporte a escala regional y muy particularmente, a escala nacional, como así también un importante centro de servicios. En el mismo sentido, Schroeder considera que "su localización, así como la disponibilidad de materias primas, facilidades educativas en todos los niveles, centros de investigación, núcleos comerciales, centros asistenciales especializados y actividades profesionales, han posibilitando el asentamiento de importantes empresas relacionadas con la actividad portuaria, agroindustrial, refinerías de petróleo y petroquímicas" (2010:2) así como le ha permitido "ser sede de organismos públicos y privados nacionales y provinciales" (Cravacuore, Ilari y Villar, 2004:71). A su vez este partido presenta un alto nivel de desarrollo humano ${ }^{17}$. En efecto, según los últimos datos referidos al Índice de Desarrollo Humano a nivel

17 El Índice de Desarrollo Humano de la Provincia de Buenos Aires incorpora indicadores alternativos debido a la indisponibilidad de algunos de los establecidos por el Programa de las 
provincial (2009-2010) el municipio de Bahía Blanca posee un IDH de 0,887, lo cual significa que estos municipios se encuentran posicionados entre los de nivel alto de desarrollo humano en la provincia, por encima de la media provincial (IDH 0,854).

\section{Aspectos socio-económicos}

Por su parte, el perfil productivo Bahía Blanca es agrícola-ganadero, orientado a la exportación, y petroquímico. Desde fines de la década de 1970, y durante los años 1980, se manifestaron "dos procesos paralelos de fuerte impacto para la ciudad: el primero de ellos sentó las bases para el desarrollo industrial del sector químico y petroquímico con la radicación de la planta de Petroquímica Bahía Blanca (1981) y por otro la creación del el Polo Petroquímico que, aunque nació esencialmente para sustituir importaciones, logró que sus plantas comenzaran a exportar a partir del año 1993” (Unión Industrial Bahía Blanca, 2010). Por otro lado, se destaca el rol de la ciudad de Bahía Blanca por su variada prestación de "servicios urbanos especializados, en particular educación y salud, así como en la jerarquización de las actividades económicas que tienen su principal componente en el complejo portuario-industrial, de relevancia nacional e internacional" (Prieto, Schroeder y Formiga, 2011:6).

En relación al sector industrial, es de destacar que de acuerdo a la Encuesta realizada por el Observatorio PyME en 2007, el 53\% de las PyME relevadas del Suroeste de la Provincia de Buenos Aires tiene su principal local industrial en el Partido de Bahía Blanca. Igualmente, este municipio “explica poco más del 50\% del Producto Bruto Geográfico (PBG) para el conjunto de los sectores; y casi el 70\% del PBG industrial, para el año 1993" (Observatorio PyME Regional Suroeste de la Provincia de Buenos Aires, 2008:23). No obstante, en términos de su perfil de inserción internacional el mismo observatorio confirma que apenas el 4\% de las PyME industriales del Suroeste de Buenos Aires destinaban más del 5\% de sus ventas hacia mercados externos para el año 2006, un promedio parecido al de la región Centro donde se ubica Tandil con un 5,2\%, mientras que el promedio provincial era del 21\%. En ese sentido, el análisis del PBG deja en evidencia que actualmente la industria manufacturera es la actividad principal, generando más del $20 \%$ del

Naciones Unidas (PNUD) en sus informes sobre Desarrollo Humano. Los actualmente utilizados son: Índice estimado de salud, de educación y de nivel de vida. Los valores del Índice van de 0 a 1, generando tres escalas: inferior a 0,5, bajo nivel de Desarrollo Humano; 0,5 a 0,8 nivel medio y superior a 0,8 nivel alto. 
producto bruto geográfico total del partido, y el 7\% de la actividad industrial de la Provincia (Observatorio PyME Regional Suroeste de la Provincia de Buenos Aires, 2008).

\section{Tabla 2.}

Producto Bruto Geográfico desagregado. Partido de Bahía Blanca. 2003

\begin{tabular}{|c|c|c|c|c|}
\hline & Sector de actividad económica & Miles de\$ & Estructura & $\begin{array}{c}\text { Participación en } \\
\text { PBG. }\end{array}$ \\
\hline A & Agricultura, ganaderia, caza y silvicultura & 33,749 & $1.2 \%$ & $\quad 0.4 \%$ \\
\hline B & Pesca y servicios conexos & 1.884 & $0.1 \%$ & $1.0 \%$ \\
\hline c & Explotación de minas y canteras & 16 & $0.0 \%$ & $0.0 \%$ \\
\hline D & Industria manufacturera & 932.303 & $32,1 \%$ & $2,3 \%$ \\
\hline E & Electricidad, gas y agua & 86.921 & $3,0 \%$ & $2,9 \%$ \\
\hline $\mathrm{F}$ & Construcción & 175,179 & $6.0 \%$ & $3.9 \%$ \\
\hline G & Comercio al por mayor, al por menor y reparaciones & 341,416 & $11,7 \%$ & $2,5 \%$ \\
\hline H & Hoteles y restaurantes & 41,400 & $1,4 \%$ & $1.7 \%$ \\
\hline 1 & Transporte, almacenamiento y comunicaciones & 330,755 & $11.4 \%$ & $2.5 \%$ \\
\hline J & Intermediación financiera y otros servicios financieros & 88.157 & $3,0 \%$ & $3,3 \%$ \\
\hline$k$ & Servicios inmobiliarios, empresariales y de alquiler & 402.890 & $13,9 \%$ & $2,3 \%$ \\
\hline L & Administración pública, defensa y reguridad social obligatoria & 158.668 & $5,5 \%$ & $3,5 \%$ \\
\hline M & Enseñanza & 111.148 & $3,8 \%$ & $2.5 \%$ \\
\hline N & Servicios sociales y de salud & 111.515 & $3,8 \%$ & $3,2 \%$ \\
\hline 0 & Servicios comunitarios, sociales y personales n.c.p. & 64.141 & $2,2 \%$ & $1,5 \%$ \\
\hline P & Servicios de hogares privadoe que contratan servicio doméstico & 28.476 & $1,0 \%$ & $2,2 \%$ \\
\hline & Total & 2.908 .618 & $100,0 \%$ & $2,3 \%$ \\
\hline
\end{tabular}

Fuente: Dirección Provincial de Estadisticas. Gobiemo de la Provincia de Buenos Aires. 2007.

Según Gárriz y Formiga (2010:4), tres sectores dinamizan la economía local:

a. El Complejo Portuario ${ }^{18}$ : es un nodo importante de transporte, que forma parte de una red multimodal permite el desarrollo de actividades productivas, al promover la radicación de emprendimientos industriales en la zona. Entre empresas relacionadas al ámbito portuario se encuentran algunas de primer nivel en el orden mundial (Cargill y Glencore-Moreno, Compañía Mega y Profertil). En 2007, se exportaron desde el Puerto de Bahía Blanca granos, aceites, inflamables y productos petroquímicos.

${ }^{18}$ El complejo portuario de Bahía Blanca está constituido por el muelle comercial de Puerto Rosales, por el Puerto Belgrano, la base Naval más importante de la Armada Argentina, por el Puerto Ingeniero White, con las instalaciones para la carga de cereales y subproductos, principal rubro de exportación del puerto, por el Puerto Galván con sus muelles de cereales y en su extremos Oeste la Terminal para combustibles líquidos y gaseosos. Bahía Blanca que es el único puerto de aguas profundas del país. De aquí salen los grandes buques de exportación de cereales, oleaginosos y subproductos (Gárriz y Formiga, 2010). 
b. El Polo Petroquímico: convierte a Bahía Blanca en uno de los centros petroquímicos destacados a nivel internacional en particular del Mercosur, principal destino de sus exportaciones, con fuerte incidencia sobre Bahía Blanca y su zona de influencia. Las empresas del Polo (fundamentalmente PBB Polisur y Solvay Indupa) incrementaron en más de un 50\% su aporte a la economía local entre 2002 y 2009.

c. El Parque Industrial ${ }^{19}$ : 42 las empresas localizadas en funcionamiento, que generan aproximadamente 1000 puestos de trabajo (Observatorio PyME Regional Suroeste de la Provincia de Buenos Aires, 2008). Entre las actividades que se desarrollan son aislaciones térmicas, construcción de intercambiadores, elaboración de artículos de limpieza, fabricación de acumuladores de plomo- ácido, cerramientos de aluminio, productos químicos y envases de polietileno y plásticos.

En los últimos años se ha buscado impulsar el sector turístico, por lo que el mismo se encuentra en crecimiento. En el caso de Bahía este objetivo busca realizarse a partir del estímulo del turismo recreativo pero principalmente tomado como punto de contacto de diferentes destinos turísticos de las localidades cercanas (sector serrano en el Partido de Tornquist y localidades atlánticas en los partidos de Monte Hermoso y Coronel Rosales) y centro de estadía, por su oferta comercial (centros comerciales y shoppings centres), la realización de numerosos congresos y convenciones y la variada actividad cultural y deportiva (Schroeder, 2010). Según la encuesta de ocupación hotelera elaborada por el INDEC, Bahía Blanca cuenta con 65.000 plazas disponibles de diferentes categorías (INDEC, 2011). De esta manera, y sin ánimos de exhaustividad, los aspectos caracterizados, revelan la existencia de importantes recursos del territorio, económicos-

${ }^{19}$ Según el Programa Nacional para el Desarrollo de Parques Industriales en el Bicentenario de los Ministerios de Industria y de Planificación Federal de la Nación Argentina, "los Parques Industriales son predios dedicados al establecimiento de la actividad industrial cuyo objetivo es potenciar el desarrollo de la industria nacional, respetando las particularidades regionales y localizados en armonía con los planes de desarrollo urbanos locales y el medio ambiente. Poseen una oferta de infraestructura y servicios comunes, favoreciendo el desarrollo de pequeñas y medianas empresas en el territorio nacional así como la generación de empleo genuino" (parques.industria.gob.ar/). 
productivos, físicos educativos y científico-tecnológicos, que proveen un contexto realmente facilitador para el desarrollo de una política de vinculación científico-tecnológica.

\section{Dimensión organizacional-administrativa}

Orientación Estratégica: Proyecto institucional y Planes estratégicos

Las cuestiones relacionadas a la vinculación se encuentran explicitadas en el Estatuto y en los planes institucionales (Plan Institucional 2005-2007 y Plan Estratégico 2011) que aluden al tema de la inserción y proyección de la universidad al medio como un eje prioritario de la gestión institucional. En particular, en el Plan Estratégico del año 2011 se propuso un eje especialmente dedicado al tema de la proyección de la universidad a partir de la coordinación de políticas conjuntas con las demás universidades de la región sobre temas estratégicos de relevancia regional. Existiendo en la región circundante de Bahía Blanca cinco universidades públicas (Universidad Provincial del Sudoeste, la Facultad Regional Bahía Blanca de la Universidad Tecnológica Nacional y las Universidades Nacionales de La Pampa, Río Negro y del Centro de la Provincia de Buenos Aires) el plan plantea una iniciativa de coordinación de políticas conjuntas sobre temas estratégicos de relevancia regional (Punto 13.4.1). Bajo esta misma línea también se impulsó el desarrollo de investigación orientada a cubrir demandas concretas de la región y el establecimiento de programas interdisciplinarios destinados al desarrollo local y a la vinculación tecnológica.

En ese mismo plan, se definió la región de incumbencia de la universidad que incluye el sudoeste de la provincia de Buenos Aires, o si se prefiere, la región contenida en un círculo centrado en la ciudad de Bahía Blanca con un radio cercano a los trescientos kilómetros, que el plan denomina región local de incumbencia.

\section{Incentivos financieros para la vinculación}

En relación a los recursos financieros que se destinan al desarrollo de la vinculación se puede señalar que la Secretaría General de Ciencia y Tecnología tuvo un rol central a partir de distintos cambios en la asignación de recursos para el desarrollo de la investigación y la transferencia de conocimiento. Como así lo señaló un funcionario de la universidad:

\section{(...) "la UNS destinó a partir del año 2004 una mayor cantidad de recursos financieros} a los proyectos de interés regional a través de la finalidad 8 del Presupuesto que a los 
proyectos convencionales"20 (ex Secretario de Ciencia y Tecnología, Universidad Nacional del Sur)

Asimismo, para dar forma a una de las políticas de inserción regional que se mencionaba en el apartado anterior, en el mismo año se abrió una convocatoria a los grupos de investigación para realizar estudios en determinadas áreas temáticas de interés local-regional que aún hoy se sostiene como parte de los lineamientos de la política de vinculación científico-tecnológica de la universidad. Esta iniciativa denominada PRI (Proyectos de Interés Regional) fue la primera propuesta de financiación concreta que marcó el inicio de una política llevada adelante por la universidad para direccionar parte de los fondos de ciencia y tecnología a ese fin.

\section{Estructuras de interacción}

En cuanto a las estructuras de interacción a través de las cuales se desarrollan las políticas de vinculación, en el año 1989 la universidad creó una fundación denominada Fundación de la Universidad Nacional del Sur (FUNS) que funcionó como enlace entre la universidad y mundo productivo. Al momento de creación de esta fundación los principios constitutivos estuvieron orientados a redefinir el vínculo de la universidad con la sociedad y fortalecer su integración al medio local y provincial. En ese momento se planteó como prioridad: “(...) rediseñar la oferta educativa a partir de las demandas reales que hace la sociedad y que requieren el aparato productivo para contribuir a su transformación y modernización (...)" (Informe Institucional, 2006:87).

En este marco, la FUNS se constituyó a partir de la necesidad de la institución de redefinir su vinculación y fortalecer su integración al medio local y provincial. Si bien en los primeros años dicha entidad sufrió diversas problemáticas, a fines de 1993 se aprobaron los estatutos, y obtuvo la

personería jurídica y se tramitó su reconocimiento por parte de la Secretaría de Ciencia y Tecnología de la Presidencia de la Nación como Unidad de Vinculación Tecnológica (UVT) condición establecida por la Ley 23877/90 de Promoción y Fomento de la Innovación Tecnológica, calidad que la habilitó para actuar conjuntamente con instituciones oficiales de investigación y el sector productivo facilitando la gestión y organización de proyectos. En este primer periodo y, a

${ }^{20} 20.000$ pesos anuales para proyectos de interés regional y 1000 para proyectos convencionales. 
través de la creación de la fundación, se intensificaron las actividades de la UNS logrando un aumento sustancial en calidad y cantidad de trabajos realizados (UNS, 2006). En este sentido, el Subsecretario señalaba que:

“(...) la FUNS era la que salía con una oferta científico-tecnológica de prestación de servicios al medio productivo, a las empresas e incluso a algún organismo del estado (...)" (ex Secretario de Ciencia y Tecnología, Universidad Nacional del Sur).

Sin embargo, a partir de 2007 con la gestión del rector Dr. Guillermo Capriste se decidió fortalecer la función de vinculación de la universidad y se avanzó en la creación de una Subsecretaría de Vinculación Tecnológica (SVT) con dependencia directa de la Secretaría General de Ciencia y Tecnología y con una posición jerárquica intermedia dentro del esquema organizacional. En su discurso de su asunción el Rector expresó que:

"la universidad debe fundamentalmente dar respuestas, a través del conocimiento, a las demandas y las necesidades reales de la sociedad a la que pertenece. (Diario Bahía Blanca, 2007).

Estas dependencias de gestión de la vinculación se establecen con el objetivo de promover las capacidades tecnológicas de la universidad y la transferencia de los resultados de la investigación científica y tecnológica (www.uns.edu.ar). Además, es la que asume la representación de la UNS en las redes nacionales como la RedVITEC (Red de Vinculación Tecnológica de las Universidades Nacionales). En tal sentido, el Subsecretario de Vinculación Tecnológica señaló que desde un comienzo la Subsecretaría:

“(...) asumió un rol de ir al medio y generar una oferta en un doble sentido, por un lado tener servicios y por otro lado se empieza a generar una serie de subsidios que estaban disponibles en la SPU con el objetivo específico de establecer líneas en vinculación tecnológica pero ya con otro sentido, con una orientación de estimular el desarrollo de la innovación a nivel regional (...)" (Subsecretario de Vinculación Tecnológica, Universidad Nacional del Sur).

Por otro lado, el Subsecretario expresó que ambas estructuras comparten el espacio físico y trabajan de manera coordinada: 


\section{“(...) la FUNS es la que administra y se encarga de la facturación a terceros y la SVT es la que genera proyectos de vinculación y representa a la UNS en las distintas redes de vinculación (Subsecretario de Vinculación Tecnológica, UNS).}

En otros casos, la Subsecretaría coordina y organiza sus actividades con organismos externos $^{21}$ como la Fundación del Sur para el Desarrollo Tecnológico (FUNDASUR), el Polo Tecnológico y la Municipalidad de Bahía Blanca.

\section{Recursos Humanos}

En relación a las características de los recursos humanos empleados en las dependencias encargadas de la función de vinculación se pueden señalar varios aspectos. En primer lugar, estas áreas generalmente son pequeñas en cuanto a cantidad de personal empleado, en general los equipos están formados por 3 a 5 personas (Llombera 2009, Carullo 2010). En el caso de la UNS, la Subsecretaría se compone de 1 Responsable de Propiedad Intelectual y Transferencia (personal contratado), 1 Responsable en I + D (personal contratado); 1 Consultor en Gestión de Proyectos (personal contratado) y 1 Asistente Administrativo (personal de planta). En relación a su formación académica se destaca la presencia de carreras de base como Agronomía (Ing. Agrónomo), Economía (Lic. en Economía) y Derecho (Abogado). Asimismo, tres de los cuatro miembros del equipo de trabajo poseen formación de posgrado (master y doctorados) y dos de ellos han realizado especializaciones vinculadas a la temática en cuestión (Master en Propiedad Intelectual y Master en Gestión Tecnológica). En este sentido, el Gerente de Vinculación Tecnológica destacó que:

\section{“(...) se promovió la formación de los recursos humanos a partir del otorgamiento de becas por parte de la propia universidad (...)" (Gerente de Vinculación Tecnológica, Universidad Nacional del Sur).}

En este marco, podemos señalar que al constituir una función nueva de la universidad en un primer momento los recursos humanos no poseían una formación específica en gestión de la vinculación, sin embargo, esta situación fue cambiando a medida que se amplió la oferta de

${ }^{21}$ Ejemplo de ello, es el concurso "Creando mi empresa" organizado por la SVT y el Polo Tecnológico de Bahía Blanca que busca estimular el emprendedorismo entre los estudiantes de la UNS o las jornadas “24 hs de innovación” organizadas por FUNS, el Polo Tecnológico y la SVT. 
formación en la temática en el país. Actualmente, podemos encontrar diversas maestrías, especializaciones y cursos de formación dictados por universidades nacionales ${ }^{22}$ orientados a formar recursos humanos especializados en gestión y vinculación tecnológica. En algunos casos, dichas especializaciones fueron financiadas por el MINCYT a través del Programa de Formación de Gerentes y Vinculadores Tecnológicos creado en 2008.

\section{Dimensión relacional}

Tipo de relacionamiento con actores públicos y privados

En relación a la forma en que la Universidad del Sur se articula con otros actores públicos y privados para el desarrollo de actividades de vinculación y su intervención en mecanismos participativos involucrados en la promoción de la innovación se puede señalar los siguientes aspectos. En el tipo de vínculos que establece con actores locales públicos y privados locales se observa una diversificada participación de empresas individuales (del rubro productos químicos), asociaciones empresariales (Asociación Industrial Química Bahía Blanca y Unión Industrial Bahía Blanca), entidades estatales (Municipio de Bahía Blanca y Coronel Rosales) y organismos del sistema científico tecnológico (INTI y FUNDASUR) y de organizaciones mixtas como el Polo Tecnológico Bahía Blanca, el Consorcio de Cooperación público-privado Tecnópolis del Sur y la Agencia Municipal de Ciencia y Tecnología ${ }^{23}$. Estos actores trabajan con la universidad de manera individual y grupal, y esta última se desarrolla a través de espacios de articulación público-privada.

\section{Participación en redes y asociaciones locales}

Por otra parte, puede verificarse la existencia de mecanismos participativas y la búsqueda de espacios de articulación entre actores públicos y privados involucrados en el proceso de innovación. Un ejemplo es el Polo tecnológico de Bahía Blanca el cuál es una asociación civil sin fines de lucro, constituida en el mes de Agosto de 2006. Está integrado por las siguientes

22 Especialización en Gestión y Vinculación Tecnológica (UNL), Especialización en Gestión de la Tecnología y la Innovación (UNSAM), Diplomatura en Gestión de la Innovación y Negocios Tecnológicos (UNICEN), Postgrado Especialización en Gestión de la Tecnología y la Innovación (UNMDP).

${ }^{23}$ La Agencia es un organismo desconcentrado dependiente de la Municipalidad de Bahía Blanca, cuya misión es diseñar e implementar políticas públicas para establecer en Bahía Blanca un modelo de desarrollo local y regional basado en el conocimiento científico y tecnológico. 
instituciones: Asamblea de Pequeños y Medianos Empresarios Regional Bahía Blanca, Corporación del Comercio Industria y Servicios de Bahía Blanca a través de la Cámara de Informática del Sur, Ente Zona Franca, FUNDASUR, Unión Industrial de Bahía Blanca, Universidad Nacional del Sur y Municipalidad de Bahía Blanca, quien tiene a cargo la presidencia. El trabajo en conjunto de todas las instituciones antes señaladas constituyen el rasgo distintivo del Polo Tecnológico Bahía Blanca. Asimismo, otro espacio lo constituye el consorcio de cooperación público-privado Tecnópolis del Sur -primer parque científico y tecnológico de Argentina en el área de electrónica- cuyo objetivo es el diseño y fabricación de circuitos integrados y productos electrónicos asociados, y brindar soporte a los actores privados de la región en sus proyectos tecnológicos. En esta iniciativa participa la Universidad Nacional del Sur, la Unión Industrial Bahía Blanca (UIBB), Zona Franca Bahía Blanca-Coronel Rosales, el Instituto Nacional de Tecnología Industrial (INTI), y distintas empresas de Base Tecnológica (ETB) de la región. Por otra parte, la UNS también forma parte de la Agencia Municipal de Ciencia y Tecnología de Bahía Blanca. Esta Agencia creada en 2011 es un organismo desconcentrado dependiente de la Municipalidad de Bahía Blanca. Su misión es diseñar e implementar políticas públicas para establecer en Bahía Blanca un modelo de desarrollo local y regional basado en el conocimiento científico y tecnológico, articulando con todos los actores públicos-privados relevantes. Breitenstein indicó que:

“(...) esta Agencia constituye un modelo público-privado de gestión donde todas las instituciones vinculadas al área de ciencia y técnica están representadas ad honorem, así como el sector privado (...)" (Discurso Intendente Bahía Blanca, 11/08/2011).

Por su parte, el Rector de la Universidad Nacional del Sur manifestó que:

“(...) consideramos muy positivo el involucramiento del Gobierno Municipal, porque uno accede a distintos mecanismos de promoción a nivel nacional pero no siempre lo puede integrar con las problemáticas locales (...)" (Discurso Rector UNS).

Dependiendo del tipo de estrategia a implementar, la UNS se relaciona de forma colaborativa con organismos como la Fundación del Sur para el Desarrollo Tecnológico (FUNDASUR), el Polo Tecnológico y la Municipalidad de Bahía Blanca. Por ejemplo, las Jornadas de Emprendedores Tecnológicos (JET) se organizan de forma conjunta entre el Polo y la Universidad del Sur. Además, 
es importante señalar la participación del Subsecretario en agencias locales y provinciales facilitando el acceso a oportunidades de innovación y articulando acciones con otros actores. En particular, el Subsecretario forma parte de la comisión directiva del Polo Tecnológico Bahía Blanca (www.ptbb.org.ar) y es el Responsable de Propiedad Intelectual y Transferencia del equipo directivo de la empresa público-privada SAPEM en el marco del consorcio Tecnópolis del Sur.

\section{Vínculos con organismos de promoción de la vinculación}

También debe mencionarse la vinculación que se establece con agencias del plano políticoinstitucional del ámbito provincial. En este sentido, se mantenían contactos directos con el Ministro de la Producción, Ciencia y Tecnología de la Provincia de Buenos Aires, Dr. Cristian Breitenstein. El ex Ministro desempeñó el cargo de intendente interino de Bahía Blanca desde abril de 2006 y luego asumió oficialmente a cargo del ejecutivo en el periodo 2007-2011. Desde el año 2011 ocupó el cargo de Ministro de la Producción, Ciencia y Tecnología del gobierno de la Provincia de Buenos Aires. Asimismo, el ex vice-rector de la Universidad Provincial del Sudoeste (UPSO) de Bahía Blanca, el Dr. Hernán Vigier también ocupó en el periodo bajo estudio un cargo bajo la órbita del Ministerio como Subsecretario de Pequeña, Mediana y Microempresa. Por otro lado, se registran vínculos asiduos con la Comisión de Investigaciones Científicas de la Provincia de Buenos Aires (CICPBA) principalmente, frente a la generación y el desarrollo de asociaciones estratégicas ${ }^{24}$ y el financiamiento de proyectos de investigación y transferencia vinculados a áreas de interés provincial $^{25}$. Su rol principal es la formación de recursos humanos dedicados a investigación en el ámbito provincial como así también el financiamiento de proyectos de innovación tecnológica. Asimismo, se tenían vínculos directos con el Vicepresidente de la Comisión de Investigaciones Científicas de la Provincia de Buenos Aires (CIC) que anteriormente había ocupado el cargo de Secretario de Ciencia y Tecnología del rectorado de la UNS. En el plano nacional, se manifiesta una activa relación con instituciones del sistema científico tecnológico como SPU, Ministerio de Ciencia, Tecnología e Innovación Productiva de la Nación, Agencia Nacional de Promoción

${ }^{24}$ La UNS creó una empresa de base tecnológica junto a organizaciones del sector público-privado, el CONICET y la Comisión de Investigaciones Científicas de la provincia de Buenos Aires (CIC).

${ }^{25}$ En el año en curso se financiaron 12 proyectos de la UNS sobre la cadena de valor apícola dentro del "Plan Estratégico Productivo Buenos Aires 2020", por tratarse de investigaciones en áreas de interés para la provincia. 
Científica y Tecnológica (ANPCYT) y CONICET en el marco de distintos proyectos y convocatorias de financiamiento a investigación y desarrollo como así también en el marco de subsidios para la creación de asociaciones estratégicas entre universidad y sector productivo.

\section{Síntesis de las capacidades institucionales universitarias desarrolladas por la UNS}

Luego de haber identificado las distintas capacidades institucionales universitarias implicadas en el desarrollo de la política de vinculación científico-tecnológica de la UNS, en primer lugar, se puede señalar que dicha universidad desarrolló distintos cambios organizacionales con la finalidad de abordar la función de vinculación. Entre ellos, se destaca la creación de una estructura de interacción ad hoc que cumple la función de enlace entre el entorno y la universidad; y la implementación de una política de incentivos para diversificar las fuentes de financiamiento como así también la asignación de fondos para promover la participación de la comunidad académica en las actividades de vinculación. En principio, se observan planes y proyectos institucionales transversales que abordan distintos aspectos de la gestión y la orientación de sus actividades de docencia e investigación con una mirada estratégica que promueve la proyección de las capacidades científico-tecnológica de la universidad hacia el entorno. En dichos planes, se propone el impulso de la investigación orientada a cubrir las demandas concretas de la región y se define un eje especialmente dedicado al tema de la proyección regional a partir de la coordinación de políticas conjuntas con otras universidades de la región. En cuanto a los incentivos para la vinculación se observa la asignación de mayores recursos a los proyectos orientados a transferir conocimiento al medio productivo que a los proyectos convencionales. En este sentido, se señala que la universidad estudiada implementó incentivos que buscaron promover los vínculos a través de cambios en las pautas de asignación de fondos hacia los grupos de investigación en contraste a incentivos que pueden otorgarse por vía salario a investigador o docente de manera individual.

En relación a las estructuras de interacción se advierte la coexistencia de dos tipos de instancias institucionales, una subsecretaría que coordina las actividades de la institución que depende de la Secretaria de Ciencia y Tecnología y un área ad-hoc descentralizada como la FUNIVEMP que actúa de soporte de las actividades de vinculación tecnológica de la universidad. En tal sentido, la fundación tuvo un rol central en los comienzos de la función de vinculación 
tecnológica en la UNS dado que las fundaciones se caracterizan por poseer mayor autonomía que las secretarías y, su condición asociativa genera mayor propensión de los actores externos para establecer proyectos con la universidad. Por último, en relación a la capacidad de dirección y liderazgo de los funcionarios encargados de las áreas se puede mencionar la formación específica en gestión de la vinculación tecnológica como así también un liderazgo activo de los funcionarios que se expresa hacia adentro y hacia fuera de la organización. En este sentido, es importante resaltar la participación de diversos funcionarios universitarios en agencias y organismos especializados locales y provinciales que les permitió proyectar las actividades de la universidad y coordinar con otros actores estrategias de actuación.

En cuanto a la capacidad relacional, la universidad estudiada llevó adelante relaciones diversificadas y articuladas con los actores locales y vínculos dinámicos con las agencias provinciales y nacionales. Respecto al primer punto, se pudo apreciar la participación de la universidad en espacios de articulación público-privado especializados en la temática (Polo y Agencia de Ciencia y Tecnología en Bahía Blanca). En cuanto a los vínculos con las agencias provinciales y nacionales se advierten relaciones dinámicas y continuas, principalmente con las agencias provinciales. La mayor vinculación establecida se basa en contactos directos a partir de la participación y liderazgo de funcionarios de la UNS y ex políticos locales en ministerios y agencias provinciales relacionadas a la promoción de la ciencia, la tecnología y la innovación. La literatura siempre aborda el liderazgo académico para el desarrollo de actividades de vinculación sin prestar la debida atención a los gestores universitarios como instrumentos importantes para la promoción de estas actividades hacia adentro y hacia afuera de la universidad. En suma, se puede señalar que el modelo organizacional de vinculación implementado por la UNS tiene un carácter descentralizado con una marcada impronta regional que le otorgó cierta flexibilidad y fluidez para entablar relaciones con los actores externos. Además, se pudo apreciar que las relaciones con la sociedad fueron motorizadas en gran medida por la participación de la universidad en espacios de articulación público y privado y los vínculos directos con funcionarios de organismos locales y provinciales especializados en la temática. 


\section{Conclusiones}

La propuesta presentada buscó contribuir en un doble sentido, teórico y empírico, a la temática en cuestión. En primer lugar, se buscó ampliar los estudios que se relacionan con la gestión de los lazos entre universidad y entorno productivo. Generalmente, los análisis ponen especial énfasis en distintos elementos internos y externos del proceso de vinculación de las universidades, sin embargo, estos aspectos siempre fueron analizados de forma separada. En tal sentido, la propuesta pretendió aportar un enfoque analítico integrado que nos permita analizar estos elementos de manera conjunta. Para ello, se introdujo un marco analítico proveniente de la ciencia política -que generalmente se utiliza para analizar las políticas públicas-como herramienta para explorar la política de vinculación pero, además como una alternativa de abordaje de la gestión de este tipo de políticas. En segundo lugar, se remarca el aporte empírico y analítico de esta investigación por aportar conocimiento sobre las universidades de tamaño mediano realizando una contribución concreta para el campo de los estudios universitarios, donde predominan los análisis focalizados en las universidades grandes o de mayor tradición científica. En tal sentido, se identificaron y analizaron las distintas capacidades institucionales universitarias implicadas en el desarrollo de la política de vinculación científico-tecnológica en una universidad argentina de gestión estatal. Con la primera dimensión, se conoció el contexto normativo-institucional y de acción, es decir las características económicas, sociales, políticas generales y particulares, que enmarcan a las universidades, realizando una aproximación a las condiciones donde los las universidades desempeñan sus actividades. Con la segunda dimensión, se amplió el análisis a las organizaciones y los actores involucrados en el desarrollo de las políticas de vinculación y el tipo de relación que la universidad establece con los actores públicos y privados. Con la tercera dimensión, se profundizó el estudio en torno a la universidad y a sus características internas, en relación al tipo de gestión, los incentivos propuestos, los recursos humanos, particularmente su capacidad de dirección y liderazgo hacia adentro y hacia fuera de la organización. Esta dimensión se combinó con el abordaje propuesto por Clark (1998) que permitió orientar el análisis hacia distintos aspectos organizacionales puntuales que complejizaron el análisis. Desde esta perspectiva multidisciplinar se concluye que en el proceso de vinculación intervienen una multiplicidad de factores, y que por lo tanto, consolidar una relación efectiva entre las universidades y los sectores productivos se vincula con un contexto determinado en el que actúa la universidad, con una relación particular que establece con su entorno y con características organizacionales específicas. Por 
último, es importante aclarar que las universidades no son iguales, insistir en la diversidad de las universidades parece superfluo pero no lo es ante la tendencia a homogeneizar lo que se consideran modelos deseables de universidades. Algunas universidades tienen un marcado ámbito local o regional en cuanto a sus intereses y proyección externa mientras otras amplían su actividad para constituirse en referentes intelectuales de carácter internacional. En este sentido, la propuesta no constituye una receta para que las universidades intervengan en estos aspectos sino analizar aquellos factores organizacionales y relacionales que se están desarrollando a nivel interno de la universidad para afrontar los desafíos que plantea un nuevo paradigma centrado en el conocimiento y la innovación. 


\section{Referencias}

Alonso, G. (2007). Elementos para el análisis de capacidades estatales. En Alonso, G. (ed.) Capacidades estatales, instituciones y política social. Buenos Aires: Prometeo Libros.

Bozeman, B. (2000). Technology transfer and public policy: a review of research and Theory. Research Policy, 29 (1), 627-655.

Casalet, M., y Casas, R. (1998). Un diagnóstico sobre la vinculación Universidad-Empresa. México: Conacyt Anuies.

Casalet, M. (2010). Mitos y realidades en la transferencia de conocimientos en América Latina, VIII Jornadas Latinoamericanas de Estudios Sociales de la Ciencia y Tecnología, Buenos Aires, Universidad Tecnológica Nacional.

Carullo, C. (2006). Indicadores de Vinculación Tecnológica en las Universidades Nacionales, Acta de la Décimo Séptima Reunión de la Comisión Ejecutiva de la Santa Fe, RedVITEC.

Chudnovsky, D., y Lopez, A. (1996). Política tecnológica en la Argentina:¿ hay algo más que laissez faire?. Redes, 3 (6), 33-75.

Clark, B. (1998). Creating Entrepreneurial Universities. Organisational pathways of transformation. Nueva York: Pergamon Press.

Clark, B. (2004). Sustaining Change in Universities, Society for Research into Higher Education. Londres: Open University Press.

Cravacuore, D., Ilari, S., y Villar, A. (2004). La articulación en la gestión local. En Cravacuore, D; Ilari, S. y Villar, A.: La articulación en la gestión municipal. Actores y políticas. Buenos Aires: Editorial de la Universidad Nacional de Quilmes.

Dagnino, R., Thomas, H., y Davyt, A. (2000). Vinculacionismo/Neovinculacionismo. Racionalidad de la interacción universidad-empresa en América latina (1955-1995). En Casas, R. y Valenti, G. (coords.), Dos ejes en la vinculación de las universidades a la producción. La formación de recursos humanos y las capacidades de investigación (pp. 25-48). México: Universidad Nacional Autónoma de México, Universidad Autónoma Metropolitana.

Decter, M., Bennett, D., y Leseure, M. (2007). University to business technology transfer- UK and USA comparisons. Technovation, (27) 3, 145-155.

D'Este, P., y Patel, P. (2005). University - Industry linkages in the UK: what are the factors determining the variety of university researchers interactions with industry?. Paper submitted to The DRUID Tenth Anniversary Summer Conference 2005, June 27-29, 2005. 
D'Este, P., y Patel, P. (2007). University-Industry linkages in the UK: what are the factors determining the variety of interactions with industry. Research Policy 36 (9), 1295-1313.

De Fuentes, C., y Dutrénit, G. (2014). Geographic proximity and university-industry interaction: the case of Mexico". The Journal of Technology Transfer, 41, (2) 329-348.

Elzinga, A. (1997). The science-society contract in historical transformation: With special reference to "epistemic drift". Social Science Information, 36 (3) 411-445.

Estebánez, M., y Korsunsky, L. (2003). Medición de actividades de vinculación y transferencia de conocimientos científicos y tecnológicos. El estado de la ciencia. Principales indicadores de ciencia y tecnología iberoamericanos. Buenos Aires: RICYT.

Evans, P. (1996). El Estado como problema y como solución. Desarrollo Económico, 35 (140).

Fernández, I., Castro, E., Conesa, A., y Gutierrez, S. (2010). Las relaciones Universidad-empresa: entre la transferencia de resultados y el aprendizaje regional. Revista Espacios, 21 (2),1-17.

Feldman, M., Feller, I., Bercovitz, J., y Burton, R. (2002). Equity and the technology transfer strategies of American research universities. Management Science, 48 (1), 105-121.

Garriz, E., y Formiga, N. (2010) Construcción de territorialidades y fragmentación socio espacial: agentes y acciones. Revista Scripta Nova, 14.

Grindle, M., y Hilderbrand, M. (1995). Building sustainable capacity in the public sector: what can be done?. Public Administration and Development, 15 (5), 441-463.

Jensen, R., Thursby, J., y Thursby, M. (2003). Disclosure and licensing of University inventions: The best we can do with the st we get to work with. International Journal of Industrial Organization, 21 (9), 1271-1300.

Kababe, Y. (2010). Las Unidades de Vinculación Tecnológica y la articulación entre el sector científico tecnológico y el sector empresario. SaberEs, 2.

Leydesdorff, L., y Etzkowitz, H. (1996). Emergence of a Triple Helix of university-industrygovernment relations. Science and public policy, 23 (5), 279-286.

Leydesdorff, L. (2012). The Triple Helix of University-Industry-Government Relations. En Carayannis, E. y Campbell, D. (Eds.), Encyclopedia of Creativity, Innovation, and Entrepreneurship. New York: Springer.

Lockett, A., Wrigth, M. y Franklin, S. (2003). Technology transfer and universities spin-out strategies. Small Business Economics, 20 (2),185-200. 
Llombera, G. (2009). Un modelo de gestión para las actividades de transferencia y vinculación tecnológica. El caso de la Universidad Nacional de Mar del Plata, Argentina. Revista Espacios, 30 (2), 29-31.

Kish, L. (1995). Representatividad, aleatorización y realismo. En Kish, L. (Comp.) Diseño Estadístico para la investigación. Madrid: Editado por CIS.

Krotsch, P. (1998). Gobierno de la Educación Superior en la Argentina: la política pública en la coyuntura (1993-1996). En Afranio Mendes Catani (org.) Novas Perspectivas nas Políticas de Educaçao Superior no Limiar do Século XXI, Campinas.

Prieto, B., Schroeder, R., y Formiga N. (2011). Ciudades intermedias dinámica y perspectivas: el caso de Bahía Blanca-Argentina. Revista Geográfica de América Central Número Especial EGAL, 1-17.

Rama, C. (2006). La tercera reforma de la educación superior en América Latina y el Caribe: masificación, regulaciones e internacionalización. Revista Educación y Pedagogía, XVIII (46), (septiembre-diciembre) 11-24.

Rubiano, M., y Rangel, P; Cuca, F. (2013). Estrategias de vinculación de los grupos de investigación con el sector productivo en el caso de la Universidad Nacional de Colombia. Equidad y Desarrollo, 20,143-165.

Siegel, D., Waldman, D., y Link, A. (2003). Assessing the impact of organizational practices on the relative productivity of university technology transfer offices: an exploratory study. Research policy, 32 (1), 27-48.

Solleiro, J., Ritter Dos Santos, E., y Escalante, F. (2000). En búsqueda de un sistema de prácticas para la vinculación exitosa de universidades y centros de $\mathrm{I}+\mathrm{D}$ con el sector productivo. Documento de trabajo, 5.

Solleiro, J. (2014). La gestión del conocimiento en las relaciones entre universidad y empresa. Informe de Investigaciones Educativas, 27 (1), 45-81.

Skopol, T. (1985). Bringing the State back in strategies of analysis in current research. En Rueschemeyer y Skocpol (comps.), Bringing the State Back in. Cambridge University Press.

Sutz, J. (2000). The university-industry-government relations in Latin America. Research policy, 29 (2), 279-290.

Vacarezza, L. (2006). Autonomía universitaria, reformas y transformación social. En Vessuri, Hebe (ed.) Universidad e investigación cientifica. Buenos Aires: FLACSO.

Versino, M., Guido, L., y Di Bello, M. (2012). Universidades y sociedades: aproximaciones al análisis de la vinculación de la universidad argentina con los sectores productivos. Buenos 
Aires: Instituto de Estudios y Capacitación, Federación Nacional de Docentes Universitarios, IEC-CONADU. 